\title{
C-terminal-truncated HBV X promotes hepato-oncogenesis through inhibition of tumor-suppressive $\beta$-catenin/BAMBI signaling
}

\author{
Seok Lee ${ }^{1,2,3}$, Mi-Jin Lee ${ }^{1,2,3}$, Jun Zhang ${ }^{1,2,3}$, Goung-Ran Yu ${ }^{1,2}$ and Dae-Ghon Kim ${ }^{1,2}$
}

C-terminal-truncated hepatitis B virus (HBV) X (HBx) (ctHBX) is frequently detected in hepatocellular carcinoma (HCC) through HBV integration into the host genome. However, the molecular mechanisms underlying ctHBx-associated oncogenic signaling have not yet been clarified. To elucidate the biological role of ctHBx in hepato-oncogenesis, we functionally analyzed ctHBx-mediated regulation of the activin membrane-bound inhibitor bone morphogenetic protein and activin membrane-bound inhibitor (BAMBI) through transforming growth factor- $\beta$ (TGF- $\beta$ ) or $\beta$-catenin (CTNNB1) in HCC cells and in an animal model, and we compared its role to that of the full-length HBx protein. Ectopic ctHBx expression generated more colonies in anchorage-dependent and -independent growth assays than did HBx expression alone. ctHBx downregulated BAMBI to a greater degree than did $\mathrm{HBx}$ in $\mathrm{HCC}$ cells. $\mathrm{HBx}$ activated the $\mathrm{Wnt} / \beta$-catenin pathway, which positively regulated the BAMBI expression through T-cell factor 1 signaling, whereas ctHBx negatively regulated the Wnt/ $\beta$-catenin pathway. BAMBI downregulated the $\beta$-catenin and TGF- $\beta 1$ signaling pathways. TGF- $\beta 1$ positively regulated BAMBI expression thorough Smad3 signaling. Furthermore, knockdown of BAMBI was more tumorigenic in HCC cells. Therefore, downregulation of both $\beta$-catenin and TGF- $\beta 1$ signaling by BAMBI might contribute to tumor suppression in mice xenotransplanted with HepG2 or SH-J1 cells. Taken together, $\mathrm{ctHBx}$ may have a more oncogenic role than HBx through its inhibition of tumor-suppressive $\beta$-catenin/BAMBI signaling. Experimental \& Molecular Medicine (2016) 48, e275; doi:10.1038/emm.2016.107; published online 2 December 2016

\section{INTRODUCTION}

Hepatocellular carcinoma (HCC) is a highly malignant tumor type and is the third most frequent cause of cancer-related death in the United States and Europe. ${ }^{1}$ A strong epidemiological correlation between chronic hepatitis B virus (HBV) infection and HCC occurrence has long been apparent. ${ }^{2}$ The HBV genome is a partially double-stranded circular DNA that is $3.2 \mathrm{~kb}$ in length and encodes genes for the $\mathrm{S}, \mathrm{X}, \mathrm{C}$ and $\mathrm{P}$ proteins. Among the four proteins translated by $\mathrm{HBV}$, the hepatitis $\mathrm{B}$ virus $\mathrm{X}(\mathrm{HBx})$ oncoprotein has been implicated in HBV-mediated hepato-carcinogenesis., ${ }^{3,4}$ During hepatocarcinogenesis, cancer cells gain an advantage through the selective reduction of the tumor-suppressive activity of transforming growth factor- $\beta$ (TGF- $\beta$ ), together with augmentation of its oncogenic activity. ${ }^{5}$ Furthermore, HBx has been reported to shift human TGF- $\beta$ signaling from tumor suppression to oncogenesis in early chronic hepatitis $\mathrm{B} .{ }^{6}$ Therefore, alterations in the TGF- $\beta$ signal transduction pathway may be involved in
HCC development. HBx mutants, particularly C-terminaltruncated mutants (ctHBx), are frequently detected in the tumor tissues of HCC patients but are rarely observed in surrounding non-tumor tissues. ${ }^{7,8}$ Furthermore, these mutant forms of HBx can also enhance the transformation abilities of ras and $m y c .^{9}$ However, little is known regarding whether or how ctHBx might be involved in carcinogenesis and tumor progression, especially in association with bone morphogenetic protein (BMP) and activin membrane-bound inhibitor (BAMBI)/TGF- $\beta$ signaling in hepato-carcinogenesis. The assumption that $\mathrm{ctHBx}$ is oncogenic through altered BAMBI/TGF- $\beta$ signaling was the basis for this study.

BAMBI, a transmembrane glycoprotein evolutionally conserved in species ranging from Xenopus to Homo sapiens, is highly homologous to TGF- $\beta / \mathrm{BMP}$ type- 1 receptors (TGFR1/BMPR1), except that it lacks an intracellular serine/threonine kinase domain. BAMBI is incorporated into complexes with TGF- $\beta / \mathrm{BMP} /$ activin type- 2 receptors

\footnotetext{
${ }^{1}$ Division of Gastroenterology and Hepatology, Department of Internal Medicine, The Research Institute of Clinical Medicine of Chonbuk National University, Jeonju, Jeonbuk, Republic of Korea and ${ }^{2}$ Biomedical Research Institute of Chonbuk National University Hospital, Jeonju, Jeonbuk, Republic of Korea ${ }^{3}$ These authors contributed equally to this work.

Correspondence: Professor D-G Kim, Division of Gastroenterology and Hepatology, Department of Internal Medicine, The Research Institute of Clinical Medicine of Chonbuk National University and Hospital, 20 Geonji-ro, Jeonju, Jeonbuk 54907, Republic of Korea.

E-mail: daeghon@chonbuk.ac.kr

Received 7 January 2016; revised 19 April 2016; accepted 11 June 2016
} 
(TGFR2/BMPR2) and, as a pseudoreceptor, antagonizes all TGF- $\beta /$ BMP and activin signaling. ${ }^{10,11}$ BAMBI is tightly co-expressed with BMP4 during embryonic development in zebrafish, Xenopus, birds and mice ${ }^{10,12-14}$ through TGF- $\beta^{15}$ and Wnt signaling. ${ }^{16}$

Human BAMBI can also promote Wnt signaling by enhancing its interaction with the receptor Frizzled-5. ${ }^{17}$ Its elevated expression has been suggested to attenuate TGF- $\beta$-mediated growth arrest in colorectal and HCC cells ${ }^{16}$ as well as induce the growth and invasion of human gastric carcinoma cells. ${ }^{18}$ BAMBI expression is also associated with Toll-like receptor 4and lipopolysaccharide-mediated hepatic fibrosis. ${ }^{19}$ Therefore, we first assessed the relationship between ctHBx or $\mathrm{HBx}$ and $\mathrm{BAMBI} / \beta$-catenin signaling. Finally, we investigated the tumor-suppressive role of BAMBI in hepato-oncogenesis.

\section{MATERIALS AND METHODS}

\section{Ethics statement}

All patients provided informed consent for a protocol that was reviewed and approved by the institutional review board of Chonbuk National University Hospital. Written informed consent was obtained from all patients. The study using experimental animals was approved by the Institutional Animal Care and Use Committee at Chonbuk National University (CUH-IACUC-140121-12).

\section{Suppression subtractive hybridization (SSH)}

SSH was performed between paired HCC and non-HCC tissue with the Clontech PCR-Select cDNA Subtraction Kit (Clontech, Mountain View, CA, USA) according to the manufacturer's protocol for HBVassociated HCC patients using two-way subtraction. In the forward subtraction, the codes from HCC were used as the tester, and the codes from paired non-HCC tissues were used as the driver; in the reverse subtraction, the codes from the paired non-HCC tissues were used as the tester, and the codes from HCC were used as the driver. Ten nanograms of the PCR product was cloned into the pGEM-T Easy Vector plasmid (Promega, Madison, WI, USA) and transformed into competent E. coli XL2-Blue cells (Stratagene, Cedar Creek, TX, USA). Two thousand colonies were randomly selected. The plasmids were purified on Qiaprep Spin Columns (Qiagen, Hilden, Germany), and the insert sequences were determined by restriction endonuclease digestion with EcoRI. The final sequence homolog searches were performed using the GenBank (nr) and EST (dbEST) databases employing the BLASTN algorithm at the National Center for Biotechnology Information (http://www.ncbi.nlm.nih.gov/BLAST).

\section{Semiquantitative reverse transcriptase-PCR (RT-PCR)}

Genomic DNA was isolated from 50 pairs of HCCs from patients with positive serum hepatitis B surface (HBs) antigen. For PCR amplification of $\mathrm{HBx}$, sets of $\mathrm{PCR}$ primers (44 F: 5'-TCCTTTGTT

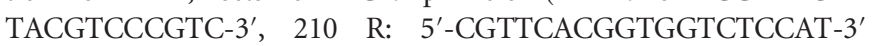
and $465 \mathrm{R}: 5^{\prime}$-TTAGGCAGAGGTGAAAAAGTTGC-3') were used for full-length and $\mathrm{COOH}$-truncated $\mathrm{HBx}$, respectively.

\section{Cloning of Enh1/Xp-ctHBx and ctHBx}

Initially, we obtained an EST clone that contained a partial HBx cDNA fused to a cDNA for the $5^{\prime}$ side of dual specificity phosphatase 26 in the chromosome 8 genomic contig. The HBx cDNA was truncated at the C-terminus by the stop codon at the junction of two genes, suggesting that a ctHBx was produced through $\mathrm{HBV}$ integration into the DNA and was functionally active in HCC. The cloning of full-length cDNAs of Enhl/Xp-tHBx was completed through two cycles of RT-PCR and overlap primer extension after cDNA amplification of the mRNA. First, $c t H B x$ was extended by searching the human HBV database; the extended sequence was confirmed using RT-PCR (primers: 5'-CACCTCCTTCCCATGGCT- ${ }^{\prime}$ and 5'-CACTT CCCCTGTAATCCC- $\left.3^{\prime}\right)$, and it contains the initiation codon. Next, Enhl/Xp was PCR-amplified using the specific primers (5'-GRAA AYTGCCTGTAAAT-3' and 5'-GGGTCGTCCGCGGGATTCAG-3'). Finally, full-length Enhl/Xp-ctHBx was obtained by overlapping primer extension using primers containing $K p n \mathrm{I}$ and $\mathrm{XhoI}$ restriction enzyme sites (5'-cggggtaccGRAAAYTGCCTGTAAAT-3' and 5'-ccgctc gagCACTTCCCCTGTAATCCC-3'), respectively, and was then ligated into pGEMT-Easy Vector (Promega). The full-length ctHBx gene was synthesized using PCR and specific primers for $H B x$ : 5'-ccggaa ttcCCTTCCCATGGCTG-3' (forward) and 5'-ccgctcgagCACTTCCC CTGTAATCCC- $3^{\prime}$ (reverse). The reaction conditions were $94^{\circ} \mathrm{C}$ for $5 \mathrm{~min}$; 40 cycles of $94^{\circ} \mathrm{C}$ for $1 \mathrm{~min}, 45-55^{\circ} \mathrm{C}$ for $1 \mathrm{~min}, 72^{\circ} \mathrm{C}$ for $1 \mathrm{~min}$; and $72^{\circ} \mathrm{C}$ for $7 \mathrm{~min}$.

\section{Luciferase reporter gene assay}

Transcriptional activation assays for the BAMBI promoter $(-255$ to $+82 \mathrm{bp}$ ) and its mutants (mt-All-Luc: mutation of all three Smad-binding element (SBE) CAG(A/C)C sequences) ${ }^{11}$ and for the TGF- $\beta 1$ promoter $(-1362$ to +11$)$ and its mutant (mutation of the $3^{\prime}$ motif of TGF- $\beta 1-D R 1)^{20}$ were conducted using luciferase reporters driven by the respective promoters. T-cell factor (TCF)-1/4-dependent transcriptional activation was also examined through a luciferase reporter gene assay using pTOP-Flash and its mutant pFOP-Flash. The dual luciferase assay was performed according to the manufacturer's instructions (Promega).

\section{Immunoblotting}

A total of $30 \mu \mathrm{g}$ of each cell or tissue lysate was separated by sodium dodecyl sulfate-polyacrylamide gel electrophoresis, and the proteins were subsequently transferred to Hybond membranes (Amersham Pharmacia Biotech, Buckinghamshire, UK). The membranes were blocked and then incubated with the designated primary antibodies, and signals were detected using an ECL Western Blotting Kit (Amersham Pharmacia Biotech). Goat anti-BAMBI polyclonal antibody (R\&D Systems, Minneapolis, MN, USA), anti-TGF- $\beta 1$ (Santa Cruz Biotechnology, Inc., Santa Cruz, CA, USA) anti- $\beta$-catenin (BD Biosciences, Franklin Lakes, NJ, USA), anti-HBx (Chemicon International, Temecula, CA, USA) and $\beta$-actin (Sigma, Saint Louis, MO, USA) were used as the primary antibodies.

\section{Immunofluorescence}

HepG2 and Hep3B cells were grown on glass coverslips, fixed in 4\% paraformaldehyde and permeabilized with $0.2 \%$ Triton $\mathrm{X}-100$ in phosphate-buffered saline. Coverslips were incubated for $1 \mathrm{~h}$ in phosphate-buffered saline containing $0.1 \%$ bovine serum albumin and a mixture of antibodies. After two washes in phosphate-buffered saline, the cells were incubated for $1 \mathrm{~h}$ with TRITC-conjugated anti-rabbit antibody (1:100 dilutions) (DAKO, Glostrup, Denmark). DNA was labeled using the Hoechst 33258 dye $\left(1 \mu \mathrm{g} \mathrm{ml}^{-1}\right)$. Cells were examined using laser scanning microscopy (LCM 510; Carl Zeiss, Jena, Germany) as previously described. ${ }^{21}$ 


\section{Anchorage-independent growth}

For the anchorage-independent growth assay, $1 \times 10^{5}$ cells were plated in $0.3 \%$ low melting point agar/growth medium onto $6-\mathrm{cm}$ dishes with a $0.6 \%$ agar underlay. After 4 weeks, colonies that were $>1 \mathrm{~mm}$ in diameter were counted.

\section{Preparation of the recombinant adenovirus}

To prepare the BAMBI-expressing adenovirus, a GFP-tagged BAMBI cDNA fragment was cloned into the KpnI and NotI sites of pENTR2B (Invitrogen, Carlsbad, CA, USA), and the GFP-tagged BAMBI insert was transferred to the $\mathrm{pAd} / \mathrm{CMV} / \mathrm{V} 5$-DEST vector (Invitrogen) by the Gateway system using LR Clonase (Invitrogen). Plasmids were linearized with PacI (Promega) and transfected into 293 A cells using Lipofectamine 2000 transfection reagent. The resulting 293A cells were cultured for 1-2 weeks in Dulbecco's modified Eagle's medium containing $10 \%$ fetal bovine serum, and the medium was replaced every 2 days. As a control, the pAd/CMV/V5-GW/lacZ vector (Invitrogen) was used to produce lacZ-bearing adenovirus.

\section{Bioluminescence imaging and analysis}

We established an orthotopic nude mouse subcutaneous tumor model with SH-J1-Luc cells overexpressing BAMBI by adenovirus delivery. Briefly, $3 \times 10^{6}$ cells in $0.08 \mathrm{ml}$ of culture medium and $0.04 \mathrm{ml}$ of $10 \%$ Matrigel were injected into the subcutaneous tissues of both shoulders of Balb/c nude mice (4 weeks old, $n=5$ ). Tumor growth was monitored once a week for 4 weeks using the Xenogen IVIS 100 Imaging System (Caliper Lifescience, Hopkinton, MA, USA; 1 min, Level B/FOV15). Mice were anesthetized with 3\% isofluorane after administration of $150 \mathrm{mg} \mathrm{kg}^{-1}$ firefly D-luciferin (Caliper Lifescience) via intraperitoneal injection for imaging.

\section{Short hairpin RNA (shRNA)-expressing lentiviral vectors and transduction}

Lentivirus vectors encoding a shRNA targeting BAMBI, $\beta$-catenin or TGFß1 (TRCN0000330319 target sequence:

\section{CCGGCTTGATCCTCAGAACTCAAATCTCGAGATTTGAGTTCTG AGGATCAAGTTTTTG, TRCN0000314991 target sequence:}

CCGGTTGTTATCAGAGGACTAAATACTCGAGTATTTAGTCCT CTGATAACAATTTTTG and the TRCN0000318388 target sequence:

CCGGCAAGCAGAGTACACACAGCATCTCGAGATGCTGTGTGTA CTCTGCTTGTTTTTG) as well as the shRNA non-specific control $(\mathrm{SHC002V})$ were used for transduction of HepG2 cells according to the manufacturer's instructions (Sigma Chemical Co). In brief, $5 \times 10^{4}$ cells were incubated overnight in a 24 -well plate and then transduced with lentiviral particles at a multiplicity of infection of 1 in the presence of $8 \mu \mathrm{g} \mathrm{ml}^{-1}$ hexadimethrine bromide. Western blotting analysis was performed to confirm specific knockdown of each vector.

\section{Statistical analysis}

The data are expressed as the mean \pm s.d. Differences were analyzed using dependent or independent $t$-tests. Values of $P<0.05$ were considered significant.

\section{RESULTS}

\section{SSH and integrated HBx expression}

Clinical characteristics of tumor and non-tumor tissues were summarized in two patients with HCC (Supplementary
Table 1). All immunohistological findings were consistent with the serological findings. Next we used SSH to monitor changes in gene expression in HCC tissues and non-tumor tissues from Case 1, as previously described. ${ }^{22,23}$ To select genes associated with HCC and functional HBx, a total of approximately 2,000 subtracted cDNA fragments were obtained from a two-way (forward and reverse) subtraction library. We identified candidate genes based on more than four appearances (Supplementary Tables 2 and 3). The digested HBV-DNA patterns showed integration of HBV-DNA in both tumor and non-tumor tissues in Case 1 (data not shown). However, the integration of HBV-DNA was not observed in tumor or non-tumor tissue in Case 2. Northern blotting analysis revealed that $H B x$ and the middle of $H B s$ mRNA were expressed in HCC (tumor) tissue but were only minimally observed in the non-tumor tissue in Case 1 (data not shown), in which an integrated stage of chronic HBV infection was observed (low HBV-DNA and positive anti-HBe $\mathrm{Ab}$ ). In contrast, neither $H B x$ nor the middle of $H B s$ was expressed in the HCC or non-tumor tissues in Case 2. Among the candidate genes, we were interested in the $B A M B I$ expressed at a low frequency in HCC because it is a negative regulator of TGF- $\beta 1 .{ }^{13,14}$

\section{Modulation of BAMBI expression by ctHBx}

HBx was detected in 50 HCC samples from 50 patients with positive HBs antigen, using PCR primers that covered 44-210 nt. However, HBx with an intact C-terminus was detected in 30 of the HCC samples (60\%), using PCR primers that covered 44-465 nt (C-terminal primer set). The remaining 20 (40\%) HCC samples without full-length HBx showed ctHBx (Supplementary Figure 1). We identified EST-F81, in which a C-terminal-truncated $H B x$ gene was fused to the $5^{\prime}$ end of dual specific phosphatase 26 from the SSH EST library (Supplementary Figure 2a). Based on this clone, we isolated and analyzed the mutations in Enh1/Xp and $c t H B x$ genes from the HCC tissues of Case 1 (Supplementary Figure $2 b$ ). Enh1/Xp contained seven nucleotide mutations, and the open reading frame of $H B x$ included 131 amino acids (aa) and seven missense mutations (Supplementary Figure 3). Notably, this ctHBX contained three missense mutations in the transforming domain (aa 1-50) and a deletion of the growth-suppressive effect domain (aa 142-154) (Figure 1a). Consistently, colony-generation assays revealed that $c t H B x$ produced greater numbers of colonies than $H B x$ or the vector control in HepG2 cells (Figure $1 \mathrm{~b}$ ), in which the $\beta$-catenin gene was known to be mutated. ${ }^{24}$ We also found that the HBx and ctHBx proteins were successfully expressed in the selected colonies (Figure 1c, left). The capacities of $\mathrm{HBx}$ and ctHBx for anchorage-independent cell growth were investigated by determining the level of cell growth as indicated by the number of colonies observed in soft agar (Figure 1c, middle and right). Similarly, $c t H B x$ produced many more colonies of HepG2 cells compared with $H B x$ or the vector control. HepG2 cells with transiently or stably expressed ctHBx exhibited inhibition of BAMBI protein expression but no change in 
a

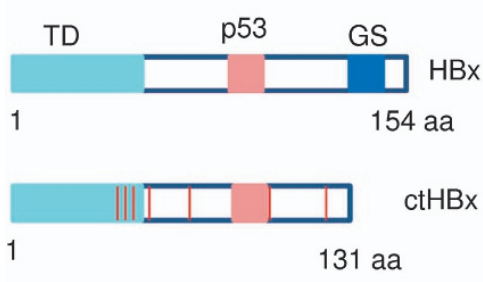

b

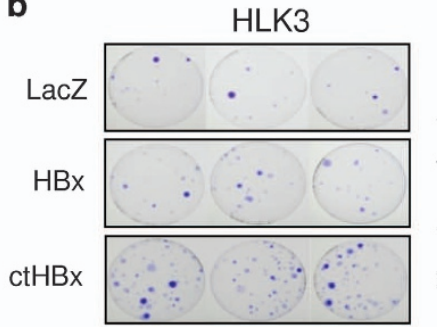

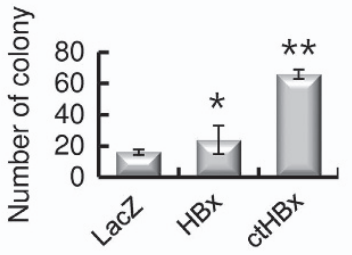

c
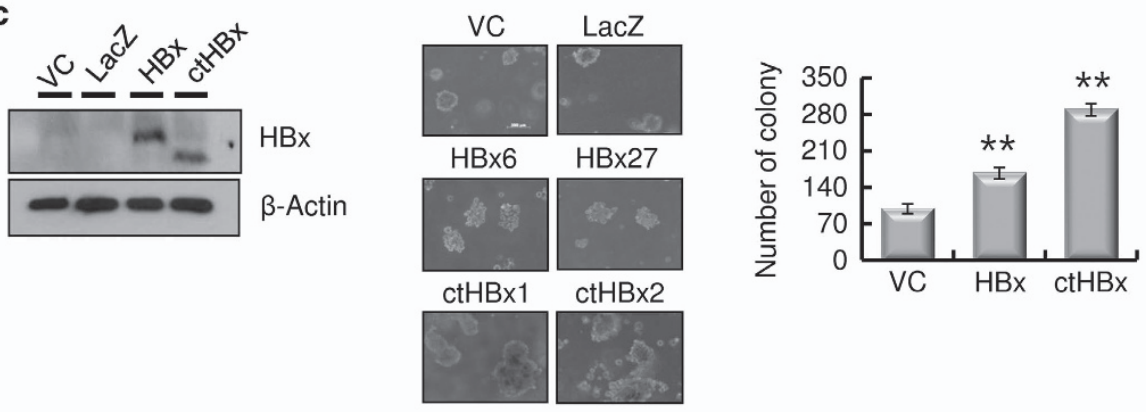

d
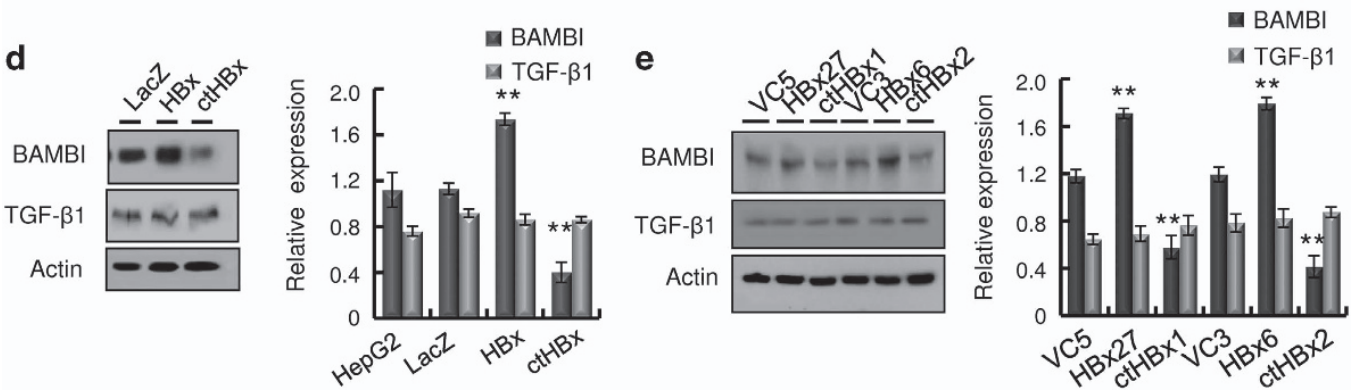

Figure 1 Regulation of BAMBI by ctHBx in HCC. (a) Putative amino-acid sequences of ctHBx. Point mutations and C-terminal deletion mutation of $\mathrm{HBx}$ (ctHBx) compared with those of wild-type HBx. TD, transforming domain (aa 1-50); p53, p53-mediated repression domain (aa 88-97); GS, growth-suppressive effect domain (aa 142-154). Red vertical lines represent mutations (lower panel). (b) Clonogenicities in the HLK3 cells after transfection with an expression vector for $\mathrm{HBx}$ or ctHBx, assessed according to the plating efficiency in a colony-forming assay. The number of colonies was visualized by crystal violet staining of the cell cultures after 14 days of G418 selection (left). Quantification of the number of colonies (right). Values represent the mean \pm s.d. from three independent experiments. ${ }^{*} P<0.05 ;{ }^{*} P<0.01$. (c) Different molecular sizes of $\mathrm{HBx}$ and ctHBx proteins in cell lysates of HLK3 cells transiently transfected with expression plasmids (left). HBx- or ctHBx-expressing cells were grown as colonies in soft agar, and the results were compared with those from the vector control (middle). The colonies shown are 15 days old. Quantification was performed in triplicate (right). The values represent the mean \pm s.d. from three independent experiments. ${ }^{*} P<0.01$. (d) Immunoblot analysis of BAMBI and TGF- $\beta 1$ protein expression in the transient transfectants expressing HBx or ctHBx in HLK3 cells (left). Quantification of the relative protein expression (right). Values represent the mean \pm s.d. from three independent experiments. ${ }^{* *} P<0.01$. (e) Immunoblot analysis of BAMBI and TGF- $\beta 1$ protein expression in stable HepG2 transfectants expressing $\mathrm{HBx}$ or ctHBx (left). Quantification of the relative protein expression (right). Values represent the mean \pm s.d. from three independent experiments. ${ }^{* *} P<0.01$.

TGF- $\beta 1$ protein expression (Figure $1 \mathrm{~d}$ and e). In contrast, the HBx-expressing HepG2 cells showed a significant increase in BAMBI expression but no change in TGF- $\beta 1$ expression. Real-time RT-PCR showed that ctHBx profoundly inhibited $B A M B I$ and TGF- $\beta 1$ mRNA expression compared with $\mathrm{HBx}$ (Supplementary Figure 4). Therefore, these results suggest that BAMBI was transcriptionally downregulated by either $\mathrm{HBx}$ or ctHBx, but it was posttranscriptionally overexpressed through an HBx-mediated alternative pathway. In addition, TGF- $\beta 1$ was transcriptionally downregulated by either $\mathrm{HBx}$ or ctHBx. However, its protein expression did not change much owing to low basal activity of the TGF- $\beta 1$ promoter.

\section{Activation of $\beta$-catenin signaling and BAMBI upregulation by $\mathrm{HBx}$, not by ctHBx}

The effects of $\mathrm{HBx}$, ctHBx and BAMBI on Wnt/ $\beta$-catenin signaling were examined using a reporter plasmid containing multimerized TCF-4-binding sites linked to the luciferase gene. BAMBI efficiently inhibited TCF-4-dependent transcriptional activation, while HBx increased this activity, and ctHBx had no effect (Figure 2a). To determine the mechanisms responsible for TCF regulation, we analyzed the subcellular localization of $\mathrm{HBx}$ and ctHBx in HepG2 cells. Immunofluorescence assays revealed that endogenous BAMBI localized prominently in the cytoplasmic membrane and less so in the nuclei of the cells, while ectopic BAMBI was expressed in the cytoplasmic 

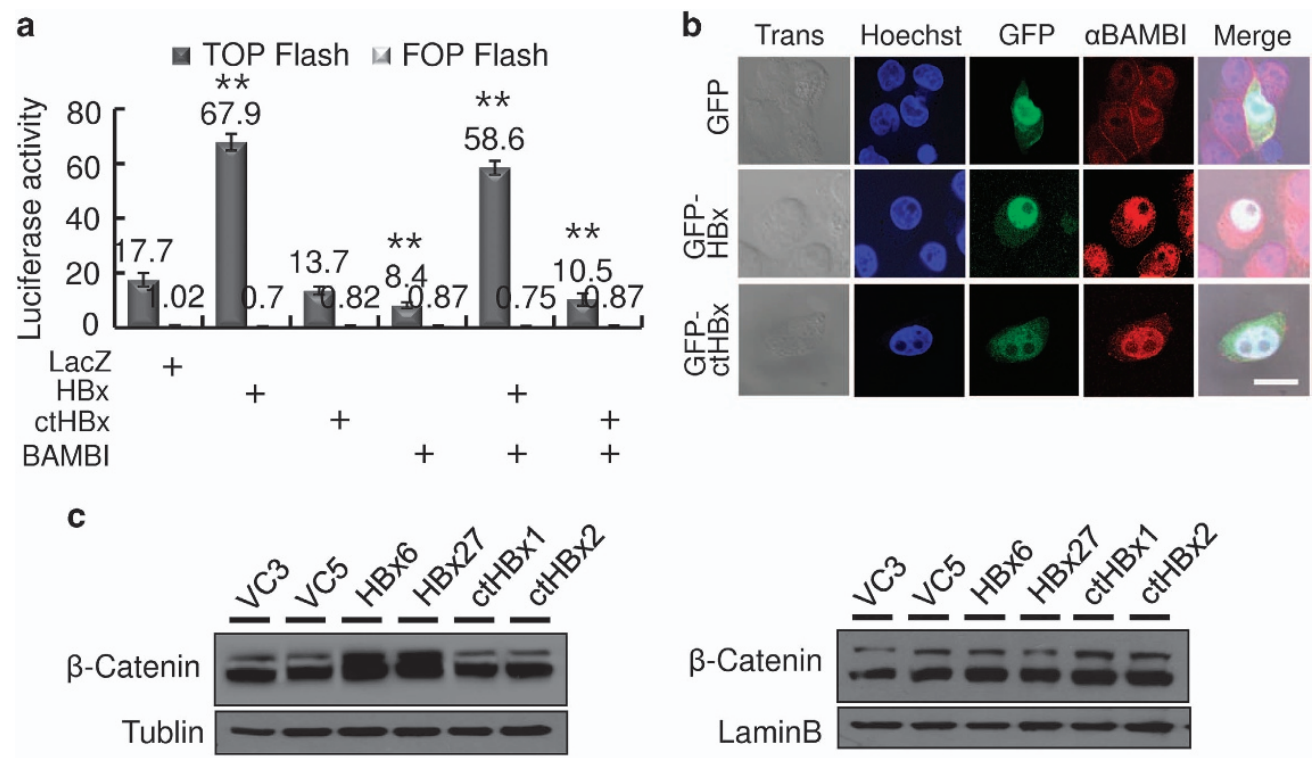

Cytoplasm

Nucleus

d
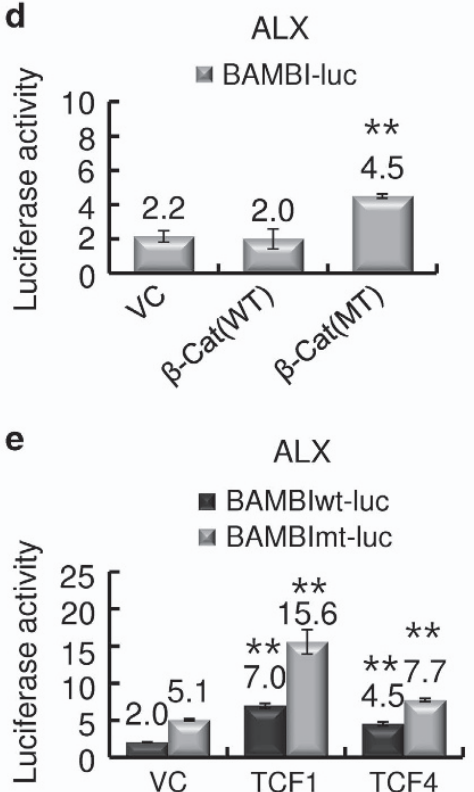

HepG2

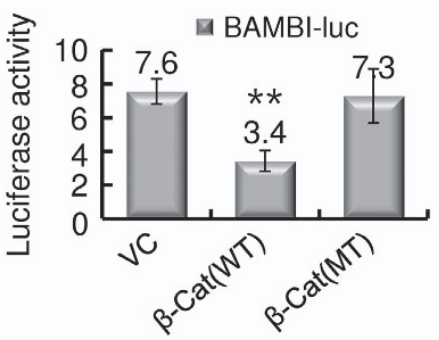

HepG2

BAMBIwt-luc

BAMBImt-luc

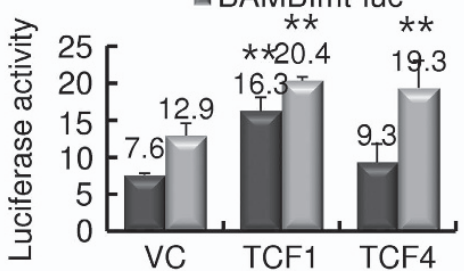

Figure 2 Modulation of Wnt/ $\beta$-catenin activation by HBx, ctHBx or BAMBI. (a) HepG2 cells were transfected with either mock (LacZ), HBx, $\mathrm{ctHBx}$ or BAMBI plasmids alone or in combination with pTOP-Flash and pFOP-Flash reporter plasmids. After $48 \mathrm{~h}$ of culture, luciferase activity was measured. The values represent the mean \pm s.d. from three independent experiments. ${ }^{*} P<0.01$. (b) Subcellular localization of $\mathrm{HBx}$ and $\mathrm{ctHBx}$ proteins. HepG2 cells were transiently transfected with GFP-tagged HBx or ctHBx, fixed in $0.4 \%$ paraformaldehyde and subsequently processed for indirect immunofluorescence with antibodies against BAMBI (TRITC, red). Nuclei were stained with Hoechst 33258 (blue), and the cells were subsequently examined using confocal microscopy (Trans, transmission; bar, $20 \mu \mathrm{m}$ ). (c) Subcellular localization of $\beta$-catenin by $\mathrm{HBx}$ or ctHBx. $\beta$-Catenin expression was determined via immunoblot analysis in nuclear or cytoplasmic fractions of the stable transfectants expressing $\mathrm{HBx}$ or ctHBx. (d) Increased BAMBI promoter activity induced by mutant $\beta$-catenin. ALX and HepG2 cells were transfected with either wild-type or mutant $\beta$-catenin expression plasmids. After $48 \mathrm{~h}$ of culture, luciferase activity was measured. The values represent the mean \pm s.d. from three independent experiments. ${ }^{* *} P<0.01$. (e) Modulation of wild-type or mutant $B A M B I$ promoter activity by TCF-1 or TCF-4 in ALX and HepG2 cells. The values represent the mean \pm s.d. from three independent experiments. ${ }^{* *} P<0.01$.

membrane of the cells (Supplementary Figure 5). Both HBx and ctHBx are abundantly localized in the nucleus and less in the cytoplasm of the cells (Figure 2b). BAMBI substantially colocalized with $\mathrm{HBx}$ and ctHBx. An immunoblot analysis showed that $\beta$-catenin accumulated in the cytoplasmic fraction in $\mathrm{HBx}$ transfectants, but less accumulation was identified in ctHBx transfectants (Figure 2c). These results suggest that cytoplasmic $\mathrm{HBx}$ appears to modulate Wnt/ $\beta$-catenin signaling. 
We also tested the effect of $\mathrm{Wnt} / \beta$-catenin signaling on the regulation of $B A M B I$ transcription. Mutant $\beta$-catenin increased the promoter activity of $B A M B I$ by more than twofold in ALX (Alexander; PLC/PRF/5) cells with wild-type $\beta$-catenin, whereas wild-type $\beta$-catenin decreased this promoter activity in HepG2 cells containing mutant $\beta$-catenin (Figure $2 \mathrm{~d}$ ). These results suggest that the basal activity of BAMBI is high owing to increased $\beta$-catenin activity; therefore, the ectopic expression of wild-type $\beta$-catenin seems to decrease the basal activity of BAMBI by competitive inhibition. Next we investigated which TCF is associated with $\beta$-catenin-mediated BAMBI expression using a luciferase reporter containing a $B A M B I$ promoter and its mutant that encoded mutated SBEs to exclude Smad-dependent BAMBI promoter activity. TCF-1 produced a greater increase in BAMBI promoter activity than did TCF-4 (Figure 2e). These results suggest that $\mathrm{Wnt} / \beta$-catenin activation increases BAMBI expression via TCF-1.

\section{Regulation of BAMBI/TGF- $\beta 1$ expression}

To evaluate the regulation of $B A M B I$ promoter activity by $\mathrm{HBx}$ or ctHBx, we transiently co-transfected HepG2 or ALX cells with LacZ, HBx or ctHBx and a BAMBI-Luc reporter construct and measured the promoter activity (Figure $3 \mathrm{a}$ ). Mock-transfected cells showed a high level of basal $B A M B I$ promoter activity in Hep G2 cells, compared with ALX cells, in which the $\beta$-catenin gene was wild type. ${ }^{24}$ This finding suggests that $\beta$-catenin activation leads to BAMBI expression. Interestingly, ctHBx expression inhibited $B A M B I$ promoter activity more profoundly than $H B x$ expression. We first introduced a mutant form of the TGF- $\beta 1$ reporter construct
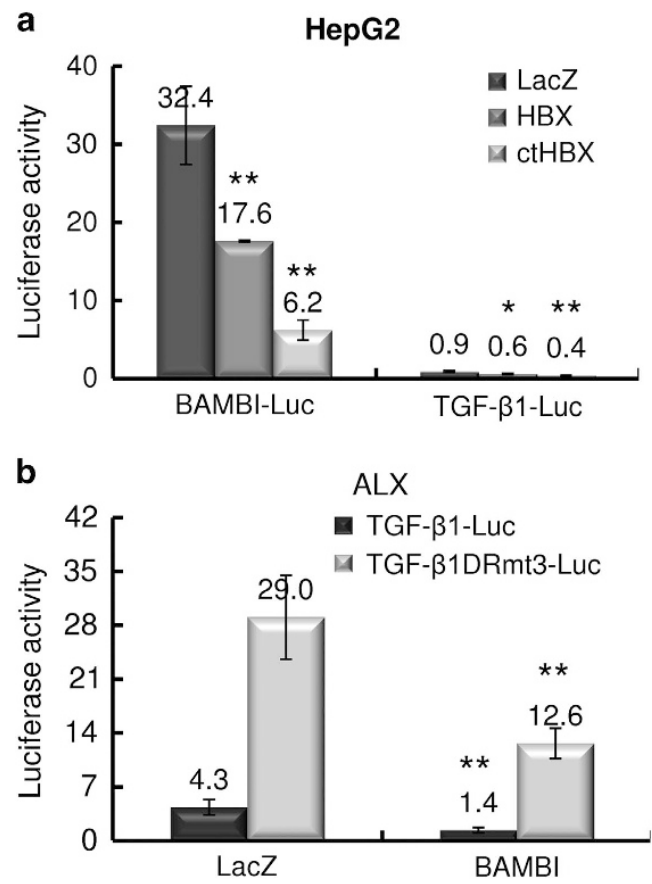

(TGF- $\beta 1$ DRmt 3 ), which contained a $3^{\prime}$ motif consisting of a direct repeat of the hexameric nucleotide sequence AGGTCA that is a peroxisome proliferator-activated receptor response element. ${ }^{20}$ Co-transfection of BAMBI resulted in a significant decrease in either wild-type or mutant TGF- $\beta 1$ promoter activity, although the basal promoter activity of wild-type TGF- $\beta 1$ was low (Figure $3 b$ ). These results imply that BAMBI efficiently suppresses TGF- $\beta 1$ signaling.

\section{Regulation of BAMBI by TGF- $\beta 1$}

Because BAMBI is known to be tightly co-expressed with BMP4 during embryonic development through TGF- $\beta 1^{15}$ and Wnt signaling, ${ }^{16}$ we next examined whether TGF- $\beta 1$ regulates BAMBI expression. Exogenous treatment with TGF- $\beta 1$ led to a dose-dependent increase in BAMBI promoter activity, with $20 \mathrm{ng} \mathrm{ml}^{-1}$ maximally stimulating TGF- $\beta 1$ promoter activity in HepG2 cells (Figure 4a). Similarly, the protein expression of BAMBI was enhanced through exogenous TGF- $\beta 1$ treatment (Figure $4 \mathrm{~b}$, upper panels). Furthermore, TGF- $\beta 1$ treatment induced a migratory and invasive morphology in the cells (Figure 4b, lower panels). To further dissect the TGF- $\beta 1$ mediated BAMBI expression, we transfected HepG2 cells singly or in combination with ctHBX, Smad2, Smad3 or Smad4 (Figure 4c, upper). Smad3 substantially increased BAMBI promoter activity, whereas Smad4 decreased this activity. An immunoblot analysis revealed similar results (Figure 4c, lower panels). In the combination experiments, Smad3 overrode the ctHBx inhibition of BAMBI promoter activity (Figure 4d), while Smad4 overrode the stimulation of Smad3. ctHBx decreased $B A M B I$ promoter activity more than $\mathrm{HBx}$.

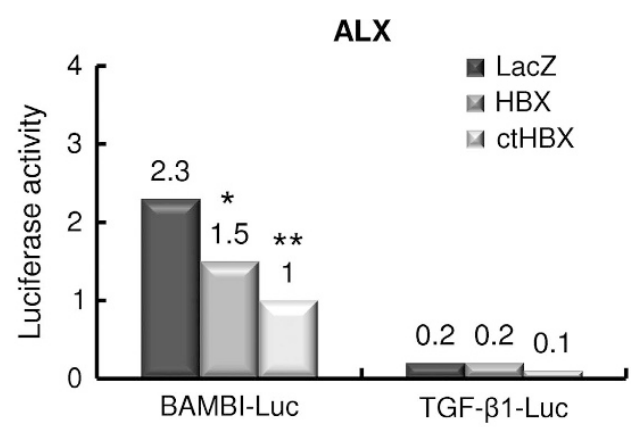


a

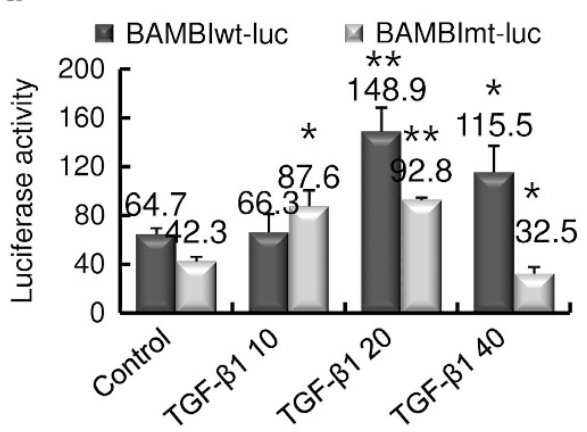

b

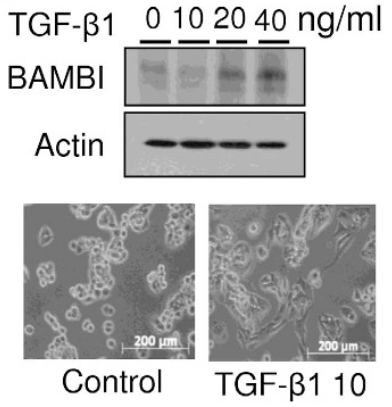

C
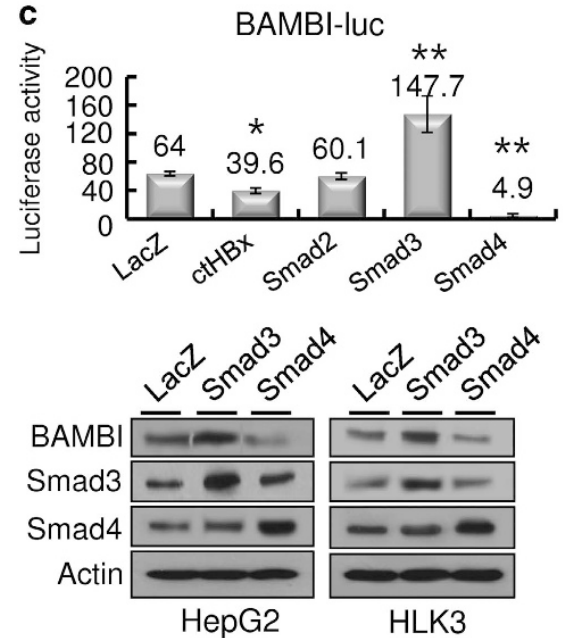

d

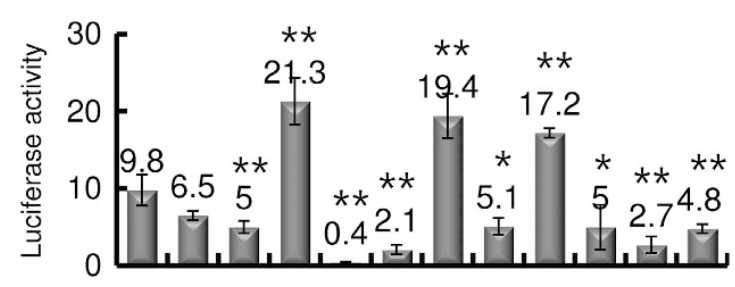

$\operatorname{LacZ}+$

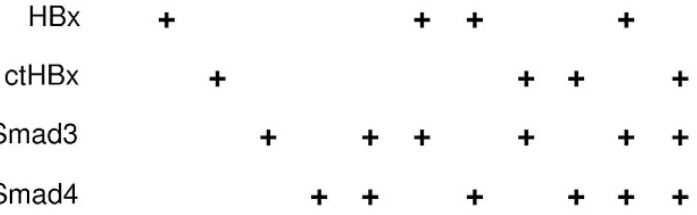

Figure 4 Regulation of BAMBI by TGF- $\beta 1$. (a) Increased BAMBI promoter activity owing to exogenous TGF- $\beta 1$ supplementation. HepG2 cells were transiently transfected with BAMBIwt-Luc $(0.5 \mu \mathrm{g})$ or BAMBImt-Luc $(0.5 \mu \mathrm{g})$ and treated with the indicated concentrations of TGF- $\beta 1$ for $48 \mathrm{~h}$. The values represent the mean \pm s.d. from three independent experiments. ${ }^{*} P<0.05$; ${ }^{* *} P<0.01$. (b) Immunoblot analysis of BAMBI protein expression in HepG2 cells treated with TGF- $\beta 1$ for $48 \mathrm{~h}$ (upper panels). Morphological changes in the HepG2 cells after treatment with exogenous TGF- $\beta 1$ (lower panels). (c) Effects of the Smad family on BAMBI promoter activity (upper panel). Regulation of BAMBI protein expression by Smad3 or Smad4 (lower panels) in HepG2 and HLK3 cells. The values represent the mean \pm s.d. from three independent experiments. ${ }^{*} P<0.05 ;{ }^{*} P<0.01$. (d) Modulation of wild-type or mutant $B A M B /$ promoter activity by ctHBx, Smad3 or Smad4, either alone or in combination. The values represent the mean \pm s.d. from three independent experiments. $* P<0.05 ; * * P<0.01$

These results suggest that TGF- $\beta 1$-mediated BAMBI expression is dependent on Smad3 expression.

\section{Inhibition of tumorigenicity by BAMBI}

Finally, we determined the functional role of BAMBI in HepG2 cells. The stable transfectants that expressed BAMBI showed morphological changes, including polygonal and epitheloid monolayer types (Figure 5a, upper panels). An immunoblot analysis of these transfectants showed that the expression of both $\beta$-catenin and TGF- $\beta 1$ was profoundly suppressed (Figure 5a, lower panels). These stable transfectants proliferated only slightly and produced small-sized colonies on soft agar compared with those produced by vector control cells (Figure 5b). In the xenotransplant mouse model, the stable transfectants hardly formed tumor masses, whereas the vector controls almost always produced tumor masses (Figure 5c). These results imply that ectopic BAMBI functions as a tumor suppressor in HepG2 cells through the strong suppression of both $\beta$-catenin and TGF- $\beta 1$. Alternatively, we examined whether BAMBI overexpression reduced tumorigenicity in vivo. A malignant HCC cell line (SH-J1) was transduced with either Ad-BAMBI/GFP or Ad-LacZ (Figure 5d, left), and colony-generation activity was examined on soft agar. SH-J1 transduced with Ad-BAMBI formed very few colonies, but control cells produced numerous colonies (Figure 5d, right lower panel). These transductants were subcutaneously inoculated into mice. Sufficient bioluminescence data were collected by 4 weeks (Figure 5e). SH-J1 cells transduced with Ad-LacZ developed large tumors $>2000 \mathrm{~mm}^{3}$ in volume within 4 weeks. However, the growth of SH-J1 cells transduced with Ad-BAMBI was markedly suppressed (Supplementary Figure 6), suggesting that BAMBI overexpression leads to significant inhibition of tumor growth in vivo. Next knockdown in HepG2 cells was established by lentiviral delivery of BAMBI, $\beta$-catenin or TGF- $\beta 1$ shRNAs (Figure $5 \mathrm{f}$, upper panels). The colony-formation ability was compared with that of cells transduced with lentiviral non-target shRNA on the soft agar (Figure 5f, lower panel). BAMBI knockdown 
cells showed more colonies than non-target control cells. In contrast, the $\beta$-catenin or TGF- $\beta 1$ knockdown cells produced fewer colonies than the non-target control cells. These results robustly support the possibility that ctHBx is more oncogenic than $\mathrm{HBx}$ through the inhibition of tumor-suppressive $\beta$-catenin/BAMBI signaling, which seems to be derived from a C-terminal truncation of $\mathrm{HBx}$ (Figure $5 \mathrm{~g}$ ).

\section{DISCUSSION}

BAMBI, a negative regulator of TGF- $\beta$ activation, is thought to have a role in hepato-oncogenesis because the expression of BAMBI is aberrantly elevated in some HCCs relative to its expression in corresponding non-cancerous tissues. ${ }^{16}$ ctHBx mutants found in HCCs have been shown to confer a selective clonal advantage in preneoplastic or neoplastic hepatocytes
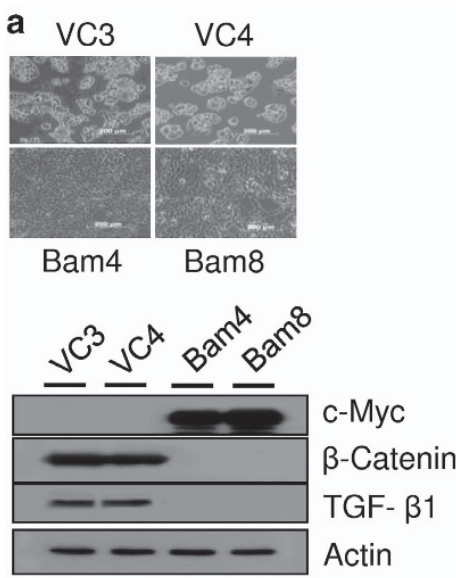

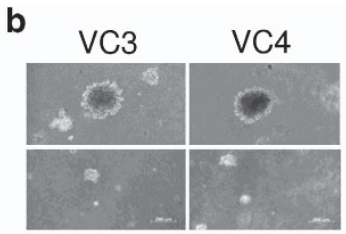

Bam4 Bam8

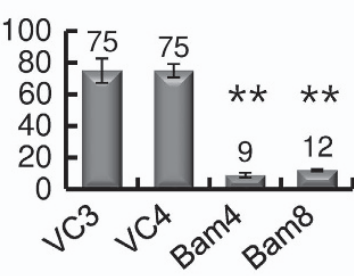

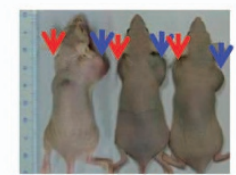

Bam4(Lt)/VC3(Rt) Bam8(Lt)/VC4(Rt)
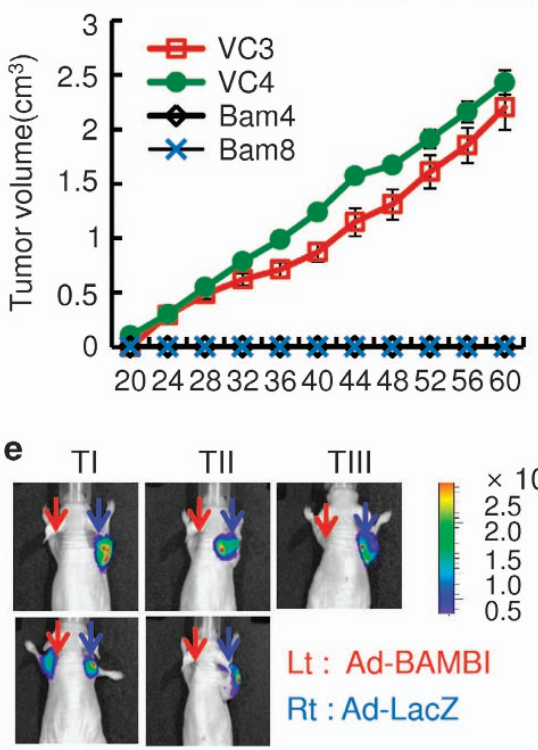

TV

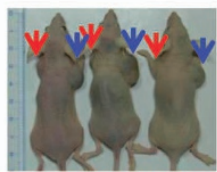

TIV

Lt : Ad-BAMB

Rt : Ad-LacZ

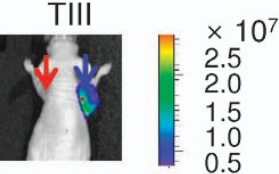

0.5
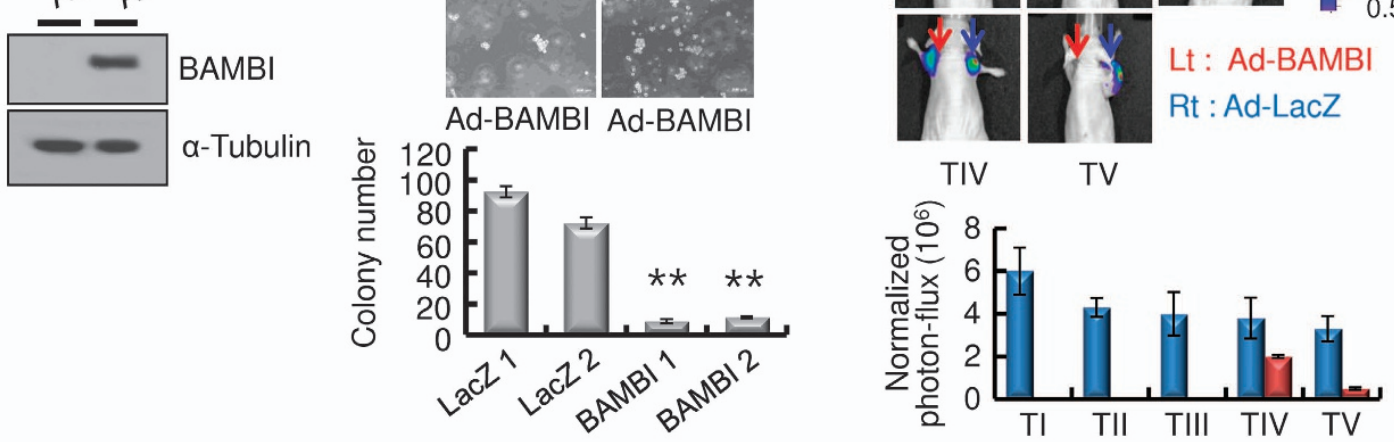

f

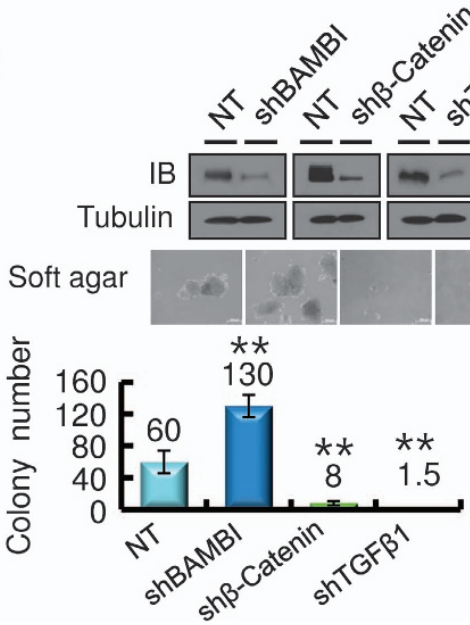

g

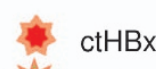

$\mathrm{HBx}$

BAMBI

TGF- $\beta 1$

$\beta$-Catenin

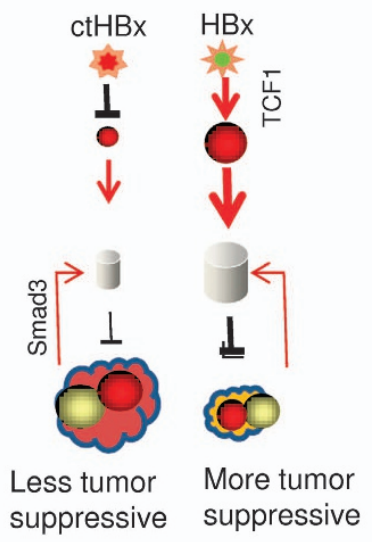


through the abrogation of p53-mediated apoptosis, which contributes to hepatocellular carcinogenesis., ${ }^{9,25}$ We speculated that functional $\mathrm{ctHBx} / \mathrm{HBx}$ modulates the expression of BAMBI and TGF- $\beta 1$, thereby influencing the development of HCC.

Our data consistently revealed that $\mathrm{ctHBx}$ overexpression enhanced the colony-forming ability and proliferative capacity in immortalized hepatocytes more than $\mathrm{HBx}$ overexpression. The transactivation activity of the C-terminal-deleted mutant of $H B x$ was analyzed using a reporter construct of CRE-Luc and a luciferase assay, and the C-terminal region of $\mathrm{HBx}$ is crucial for transcriptional function. ${ }^{9}$ The specific transcriptional activity of $c t H B x$ remains unclear; however, our data revealed that both $\mathrm{HBx}$ and $\mathrm{ctHBx}$ are abundantly localized in the nucleus and less in the cytoplasm of HepG2 and ALX cells. An immunoblot analysis showed that $\beta$-catenin accumulated in the cytoplasmic fraction in $\mathrm{HBx}$ transfectants but less in ctHBx transfectants. Cytoplasmic $\mathrm{HBx}$ appears to modulate Wnt/ $\beta$-catenin signaling. In HepG2 cells, the basal level of BAMBI activation was relatively high, while that of TGF- $\beta 1$ was low, as previously observed in other HCC cases. ${ }^{10,16}$ BAMBI is a transmembrane protein that lacks an intracellular kinase domain but has high sequence similarity to the extracellular domain of T $\beta$ RI and thus inhibits TGF- $\beta$ signaling by forming a heterodimer with T $\beta$ RII. ${ }^{10}$ However, our data revealed that BAMBI also transcriptionally regulates TGF- $\beta 1$ expression.

BAMBI is also directly induced by TGF- $\beta$ signaling through three tandem repeats of a 13-bp sequence containing SBEs. In other words, BAMBI transcription is regulated through the direct binding of Smad3 and Smad4 to its promoter. ${ }^{11}$ However, our cell culture system showed that Smad3 stimulated $B A M B I$ promoter activity, whereas Smad4 depressed it. This Smad3-mediated BAMBI expression can contribute to hepato-carcinogenesis through TGF- $\beta$ signaling, as previously reported. ${ }^{6}$ Furthermore, a mutation in the SBE abrogated the Smad3-stimulatory effect but did not change the inhibitory activity of Smad4. Therefore, the SBE in BAMBI seems to be the binding site for Smad3, but not for Smad4.
$\beta$-Catenin mutation leads to the functional activation of $\mathrm{Wnt} / \beta$-catenin signaling in hepatoma cells, and the amount of literature on the stabilizing mutation of $\beta$-catenin in HCC is increasing. ${ }^{26-28}$ In addition to mutations of $\beta$-catenin, ectopic expression of $\mathrm{HBx}$ along with Wnt-1 has also been reported to activate $\mathrm{Wnt} / \beta$-catenin signaling in Huh7 cells by stabilizing cytoplasmic $\beta$-catenin, which was achieved by suppressing glycogen synthesis kinase 3 activity via the activation of Src kinase. ${ }^{29}$ Our study showed that $\mathrm{HBx}$ activated $\mathrm{Wnt} / \beta$-catenin signaling, which in turn positively regulated BAMBI through TCF-1. BAMBI negatively modulated $\mathrm{Wnt} / \beta$-catenin signaling in HepG2 HCC cells, which differs from previous data found in HEK293T cells. ${ }^{17}$ Although, $\beta$-catenin activation increased the promoter activity of BAMBI, the deletion mutants of ctHBx and HBx decreased the promoter activity of $B A M B I$ in HepG2 and ALX cells because they have transactivation domains and regulate the transcriptional activity of other target genes in addition to $\beta$-catenin target genes. ${ }^{30,31}$ ctHBx profoundly suppressed BAMBI promoter activity owing to the lack of activated $\mathrm{Wnt} / \beta$-catenin signaling, whereas $\mathrm{HBx}$ less profoundly suppressed BAMBI promoter activity through activated $\mathrm{Wnt} / \beta$-catenin signaling. Thus our data suggest that $\mathrm{HBx}$ stimulates Wnt/ $\beta$-catenin signaling, which is followed by tumor-suppressive $\beta$-catenin/BAMBI signaling. Furthermore, ectopic overexpression of BAMBI negatively downregulated both TGF- $\beta 1$ and $\beta$-catenin signaling in HepG2 cells, which consequently appeared to be associated with the suppression of oncogenicity. Thus the induction of BAMBI may function in part as a tumor-suppressor mechanism in HCC. In contrast, ctHBx seems to be more oncogenic than HBx through the inhibition of tumor-suppressive $\beta$-catenin/ BAMBI signaling.

In conclusion, the data presented here provide a novel mechanism by which ctHBx might contribute to strong hepato-oncogenesis via the loss of tumor-suppressive $\beta$-catenin/BAMBI signaling.

Figure 5 BAMBI functions as a tumor suppressor in vitro and in vivo. (a) BAMBI-mediated morphological changes in HepG2 cells stably overexpressing the BAMBI protein (upper panels). Immunoblot analysis of $\beta$-catenin and TGF- $\beta 1$ in the stable transfectants overexpressing the c-Myc-tagged BAMBI protein (lower panels). (b) The BAMBI-expressing HepG2 cells showed minimal colony growth in soft agar compared with that of the vector control (upper panels). The colonies shown are 15 days old. Quantification was performed in triplicate (lower panel). The values represent the mean \pm s.d. from three independent experiments. ${ }^{* *} P<0.01$. (c) Growth of the tumor masses in BAMBI-expressing HepG2 cells and vector control cells injected into the left shoulders (red arrows) of nude mice. Vector control cell lines injected into the right shoulder (blue arrows), grew quickly and formed tumor masses, whereas the BAMBI-expressing cells did not (upper panels). The tumor volume was measured as a function of time (lower panel). Each value represents the mean \pm s.d. (d) BAMBI expression in SH-J1 cells infected with either Ad-LacZ or Ad-BAMBI (left). The Ad-BAMBI-infected SH-J1 cells showed minimal colony growth in soft agar compared with the parent or Ad-LacZ-infected cells (right upper panels). The colonies shown are 15 days old. Quantification was performed in triplicate (right lower panel). The values represent the mean \pm s.d. of three independent experiments. $* * P<0.01$. (e) Bioluminescent images of the subcutaneous tumor masses ( $n=5$ mice per group, representative anterior-posterior images, 1 min exposure time). SH-J1 cells infected with Ad-LacZ subcutaneously injected into the right shoulder (blue arrows) grew quickly and formed tumor masses, but the Ad-BAMBI-infected cells injected into the left shoulder (red arrows) formed rare, small tumor masses (upper panels). Quantitative measurement of photon flux (lower panel). The values represent the mean \pm s.d. (f) Silencing of TGF- $\beta 1 / B A M B I / \beta-c a t e n i n$ protein expression by lentiviral delivery of shRNA (upper panels) in HepG2 cells and their clonogenic ability in the soft agar (middle panels) and quantification (lower panel). The values represent the mean \pm s.d. of three independent experiments. ${ }^{* *} P<0.01$. (g) A schematic model of the inhibition of BAMBI signaling by ctHBx. HBx profoundly stimulates Wnt/ $\beta$-catenin signaling, and overexpression of BAMBI suppresses both TGF- $\beta 1$ and $\beta$-catenin signaling and subsequently inhibits tumorigenicity. $\longrightarrow$, inhibition; $\longrightarrow$, stimulation. 


\section{CONFLICT OF INTEREST}

The authors declare no conflict of interest.

\section{ACKNOWLEDGEMENTS}

We thank S-J Kim (Cha University, South Korea), H-G Seo (Gyeongsang University, South Korea), and T Akiyama (Tokyo University, Japan) for their generous contributions of recombinant plasmids. This study was supported by the Mid-career Researcher Program through a National Research Foundation of Korea (NRF) grant funded by the Ministry of Education, Science and Technology (MEST) (2013R1A2A1A01009354) and by the Fund of Biomedical Research Institute, Chonbuk National University Hospital. This study was also supported by the National R\&D Program for Cancer Control (0620220), Ministry for Health, Welfare and Family Affairs, Republic of Korea.

Author contributions: SL, ML, JZ and DK conceived and designed the experiments. SL, ML and GY performed the experiments. SL, ML and DK analyzed the data. SL, ML, JZ, GY and DK contributed reagents/ materials/analysis tools. SL, ML and DK wrote the paper.

1 El-Serag HB. Hepatocellular carcinoma: recent trends in the United States. Gastroenterology 2004; 127: 27-34.

2 Brechot C, Pourcel C, Louise A, Rain B, Tiollais P. Presence of integrated hepatitis $B$ virus DNA sequences in cellular DNA of human hepatocellular carcinoma. Nature 1980; 286(5772): 533-535.

3 Kim CM, Koike K, Saito I, Miyamura T, Jay G. HBx gene of hepatitis B virus induces liver cancer in transgenic mice. Nature 1991; 351: 317-320.

4 Yu DY, Moon HB, Son JK, Jeong S, Yu SL, Yoon H et al. Incidence of hepatocellular carcinoma in transgenic mice expressing the hepatitis B virus X-protein. J Hepatol 1999; 31: 123-132.

5 Roberts AB, Sporn MB. The transforming growth factor-betas: past, present, and future. Ann NY Acad Sci 1990; 593: 1-6.

6 Murata M, Matsuzaki K, Yoshida K, Sekimoto G, Tahashi Y, Mori S et al. Hepatitis $B$ virus $X$ protein shifts human hepatic transforming growth factor (TGF)-beta signaling from tumor suppression to oncogenesis in early. chronic hepatitis B. Hepatology 2009; 49: 1203-1217.

7 lavarone M, Trabut JB, Delpuech O, Carnot F, Colombo M, Kremsdorf D et al. Characterisation of hepatitis $\mathrm{B}$ virus $\mathrm{X}$ protein mutants in tumour and non-tumour liver cells using laser capture microdissection. J Hepatol 2003; 39: 253-261.

8 Wang Y, Lau SH, Sham JS, Wu MC, Wang T, Guan XY et al. Characterization of HBV integrants in 14 hepatocellular carcinomas: association of truncated X gene and hepatocellular carcinogenesis. Oncogene 2004; 23: $142-148$.

9 Tu H, Bonura C, Giannini C, Mouly H, Soussan P, Kew M et al. Biological impact of natural $\mathrm{COOH}$-terminal deletions of hepatitis $\mathrm{B}$ virus $\mathrm{X}$ protein in hepatocellular carcinoma tissues. Cancer Res 2001; 61: 7803-7810.

10 Onichtchouk D, Chen YG, Dosch R, Gawantka V, Delius H, Massagué J et al. Silencing of TGF-beta signalling by the pseudoreceptor BAMBI. Nature 1991; 401: 480-485.

11 Sekiya T, Oda T, Matsuura K, Akiyama T. Transcriptional regulation of the TGF-beta pseudoreceptor BAMBI by TGF-beta signaling. Biochem Biophys Res Commun 2004; 320: 680-684.

12 Grotewold L, Plum M, Dildrop R, Peters T, Rüther U. Bambi is coexpressed with Bmp-4 during mouse embryogenesis. Mech Dev 2001; 100: 327-330.

13 Tsang M, Kim R, de Caestecker MP, Kudoh T, Roberts AB, Dawid IB. Zebrafish nma is involved in TGFbeta family signaling. Genesis 2000; 28 : 47-57.

14 Higashihori N, Song Y, Richman JM. Expression and regulation of the decoy bone morphogenetic protein receptor BAMBI in the developing avian face. Dev Dyn 2008; 237: 1500-1508.
15 Xi Q, He W, Zhang XH, Le HV, Massagué J. Genome-wide impact of the BRG1 SWI/SNF chromatin remodeler on the transforming growth factor beta transcriptional program. Biol Chem 2008; 283: 1146-1155.

16 Sekiya T, Adachi S, Kohu K, Yamada T, Higuchi O, Furukawa Y et al. Identification of BMP and activin membrane-bound inhibitor (BAMBI), an inhibitor of transforming growth factor-beta signaling, as a target of the beta-catenin pathway in colorectal tumor cells. J Biol Chem 2004; 279: 6840-6846.

17 Lin Z, Gao C, Ning Y, He X, Wu W, Chen YG. The pseudoreceptor BMP and activin membrane-bound inhibitor positively modulates Wnt/beta-catenin signaling. J Biol Chem 2008; 283: 33053-33058.

18 Sasaki T, Sasahira T, Shimura H, Ikeda S, Kuniyasu H. Effect of Nma on growth inhibition by TGF-betaa in human gastric carcinoma cell lines. Oncol Rep 2004; 11: 1219-1223.

19 Seki E, De Minicis S, Osterreicher CH, Kluwe J, Osawa Y, Brenner DA et al. TLR4 enhances TGF-beta signaling and hepatic fibrosis. Nat Med 2007; 13: 1324-1332.

20 Kim HJ, Ham SA, Kim SU, Hwang JY, Kim JH, Chang KC. Transforming growth factor-beta1 is a molecular target for the peroxisome proliferatoractivated receptor delta. Circ Res 2008; 102: 193-200.

21 Yun BR, Lee MJ, Kim JH, Kim IH, Yu GR, Kim DG et al. Enhancement of parthenolide-induced apoptosis by a PKC-alpha inhibition through heme oxygenase-1 blockage in cholangiocarcinoma cells. Exp Mol Med 2010; 42: 787-797.

22 Lee MJ, Yu GR, Yoo HJ, Kim JH, Yoon BI, Choi YK et al. ANXA8 down-regulation by EGF-FOXO4 signaling is involved in cell scattering and tumor metastasis of cholangiocarcinoma. Gastroenterology 2009; 137: $1138-1150$.

23 Diatchenko L, Lau YF, Campbell AP, Chenchik A, Moqadam F, Huang B et al. Suppression subtractive hybridization: a method for generating differentially regulated or tissue-specific cDNA probes and libraries. Proc Natl Acad Sci USA 1996; 93: 6025-6030.

24 de La Coste A, Romagnolo B, Billuart P, Renard CA, Buendia MA, Soubrane $O$ et al. Somatic mutations of the beta-catenin gene are frequent in mouse and human hepatocellular carcinomas. Proc Natl Acad Sci USA 1998; 95: 8847-8851.

25 Wang Y, Lau SH, Sham JS, Wu MC, Wang T, Guan XY. Characterization of HBV integrants in 14 hepatocellular carcinomas:association of truncated $X$ gene and hepatocellular carcinogenesis. Oncogene 2004; 23: 142-148.

26 Miyoshi Y, Iwao K, Nagasawa Y, Aihara T, Sasaki Y, Imaoka S et al. Activation of the beta-catenin gene in primary hepatocellular carcinomas by somatic alterations involving exon 3. Cancer Res 1998; 58: 2524-2527.

27 Wei Y, Fabre M, Branchereau S, Gauthier F, Perilongo G, Buendia MA. Activation of beta-catenin in epithelial and mesenchymal hepatoblastomas. Oncogene 2000; 19: 498-504.

28 Terris B, Pineau P, Bregeaud L, Valla D, Belghiti J, Tiollais $\mathrm{P}$ et al. Close correlation between beta-catenin gene alterations and nuclear accumulation of the protein in human hepatocellular carcinomas. Oncogene 1999; 18: 6583-6588.

29 Cha MY, Kim CM, Park YM, Ryu WS. Hepatitis B virus X protein is essential for the activation of Wnt/beta-catenin signaling in hepatoma cells. Hepatology 2004; 39: 1683-1693.

30 Murakami S. Hepatitis B virus X protein: structure, function and biology. Intervirology 1999; 42: 81-99.

31 Murakami S. Hepatitis B virus $\mathrm{X}$ protein: multifunctional viral regulator. J Gastroenterol 2001; 36: 651-660.

(c) (1) This work is licensed under a Creative Commons Attribution 4.0 International License. The images or other third party material in this article are included in the article's Creative Commons license, unless indicated otherwise in the credit line; if the material is not included under the Creative Commons license, users will need to obtain permission from the license holder to reproduce the material. To view a copy of this license, visit http:// creativecommons.org/licenses/by/4.0/

Supplementary Information accompanies the paper on Experimental \& Molecular Medicine website (http://www.nature.com/emm) 